\title{
Mesenchymal stem cells overexpressing adrenomedullin improve heart function through antifibrotic action in rats experiencing heart failure
}

\author{
LI LI LI ${ }^{1,2}$, CHENG PENG $^{1,2}$, MEILING ZHANG ${ }^{3}$, YUMEI LIU ${ }^{1,2}$, HEFEI LI $^{4}$, \\ ${\text { HUIBO } \mathrm{CHEN}^{5} \text {, YU SUN }}^{1,2}$, CHUNJUN ZHU ${ }^{1}$ and YAO ZHANG ${ }^{1,2}$
}

${ }^{1}$ Department of Cardiology, The Second Affiliated Hospital of Harbin Medical University; ${ }^{2}$ The Key Laboratory of Myocardial Ischemia, Harbin Medical University, Ministry of Education; ${ }^{3}$ Department of Cardiology, The Third Affiliated Hospital, Harbin Medical University, Harbin, Heilongjiang 150086; ${ }^{4}$ Department of Cardiology, The Central Hospital of Handan, Handan, Hebei 056000; ${ }^{5}$ Department of Cardiology, The Second Hospital of Harbin, Harbin, Heilongjiang 150086, P.R. China

Received June 19, 2016; Accepted March 13, 2017

DOI: $10.3892 / \mathrm{mmr} .2017 .8049$

\begin{abstract}
Previous studies of the authors have indicated that the transplantation of mesenchymal stem cells (MSCs) can attenuate cardiac fibrosis through the secretion of antifibrotic factors, such as adrenomedullin (ADM). Therefore, the authors addressed the hypothesis that ADM overexpression could enhance the antifibrotic effect of MSCs transplantation in a rat model of heart failure. The results of the present study demonstrated that, compared with the group that received the GFP-MSCs, the transplantation of ADM-MSCs significantly improved heart function and decreased the percentage of fibrotic area and the expression of matrix metalloproteinase 2. In addition, fluorescence microscopy indicated that the survival of transplanted MSCs also increased significantly in the ADM-MSCs-treated group. Furthermore, the expression of fibrosis-related genes, such as ADM and hepatocyte growth factor, were significantly influenced in the ADM-MSCs-treated group. Based on these findings, it may be concluded that, compared with MSCs, MSCs overexpressing ADM can further improve heart function in rats experiencing heart failure through enhanced antifibrotic activity.
\end{abstract}

\section{Introduction}

Stem cell transplantation has been extensively investigated as a therapy for heart failure $(1,2)$. Mesenchymal stem cells (MSCs), which have the ability to escape detection by the host

Correspondence to: Dr Yao Zhang, Department of Cardiology, The Second Affiliated Hospital of Harbin Medical University, 256 Xuefu Road, Harbin, Heilongjiang 150086, P.R. China

E-mail: yaozhang_grace@aliyun.com

Key words: adrenomedullin, cardiac fibrosis, heart failure, mesenchymal stem cells, transplantation immune system upon transplantation and to expand easily, become a promising source of cells for transplantation (3).

Numerous studies have demonstrated that the transplantation of MSCs can promote cardiac repair in acute myocardial injury and cardiomyopathy (3-6). The mechanisms mediating the beneficial effect of MSC transplantation have been widely investigated. In a previous study of the authors, the results demonstrated that the antifibrotic effect of MSCs contributes to the improvement of heart function $(6,7)$. Despite progress, the transplantation of MSCs is insufficient to fully restore heart function. Therefore, an important issue to be solved is how to maximize the therapeutic effect of MSCs transplantation. It has been proven that gene modification can increase the efficacy of cell transplantation $(8,9)$. Adrenomedullin (ADM) is a cytokine that may modulate cardiac fibroblast growth (10-12). ADM infusion during ischemia/reperfusion has been proven to attenuate cardiac fibrosis in rats (13).

Therefore, in the present study, the aim was to examine whether ADM gene transfer can increase the therapeutic effects of MSC transplantation in isoproterenol-induced global heart failure in rats by attenuating cardiac fibrosis and to investigate the mechanisms that mediate this effect.

\section{Materials and methods}

Culture and adenoviral transduction of MSCs. MSCs were obtained and passaged according to previously described methods $(6,7)$. At the third passage, the MSCs were transduced with an adenoviral vector encoding the gene green fluorescent protein (Ad-CMV-GFP). To select the best multiplicity of infection (MOI) for the adenovirus-mediated gene transfer, MSCs were exposed to Ad-CMV-GFP at MOIs of 1:50, 1:100, 1:200 and 1:400 for $8 \mathrm{~h}$. The medium was then replaced by normal Dulbecco's modified Eagle's medium (DMEM)/F12 containing $10 \%$ fetal bovine serum and antibiotics. At $48 \mathrm{~h}$ following transfection, cell viability was determined through observation of cellular morphology by fluorescence microscopy. Based on these observations, 1:200 was chosen as the optimal MOI because of its high efficiency and low 
toxicity. Transduction efficacy was further assessed by fluorescence-activated cell sorting (FACS) analysis. For FACS analysis, the transfected MSCs were digested and centrifuged at $1,000 \mathrm{x} \mathrm{g}$ for $5 \mathrm{~min}$. Following removal of the supernatant, $500 \mu 1$ PBS was added and the MSCs were assayed by flow cytometry and analyzed using FACSDiva Pro software (BD Biosciences, Franklin Lakes, NJ, USA). In addition, ADM expression in the GFP-MSCs and ADM-MSCs was detected by western blotting.

Experimental model and group. A total of 55 male Wistar rats (180-200 g) were obtained from Harbin Medical University Laboratorial Animal Center (Harbin, China). All experimental procedures were approved by the Care of Experimental Animals Committee of Harbin Medical University (Harbin, China). Isoproterenol (ISO)-induced heart failure was induced as described previously (7). Briefly, ISO (170 mg/kg in $0.5 \mathrm{ml}$ saline; Sigma-Aldrich; Merck KGaA, Darmstadt, Germany) was subcutaneously injected into the rats every day for 4 consecutive days. Sham-injected rats were injected subcutaneously with $0.5 \mathrm{ml}$ saline alone. At 4 weeks following the final injection, these rats received transplants. Before transplantation, the animals were randomized into four groups: The sham group and three ISO-induced heart failure groups. The three heart failure groups each received a different treatment: DMEM/F12 (medium-treated group), GFP-MSCs (GFP-MSCs-treated group) or ADM-MSCs transplantation (ADM-MSCs-treated group).

Cell transplantation. At 4 weeks following the final ISO or saline injection, all of the rats received transplantation. In the GFP-MSCs or ADM-MSCs-treated groups, a total of 5x10 GFP-MSCs or ADM-MSCs were suspended in $100 \mu \mathrm{l}$ medium and injected into the left anterior myocardium through a $30-\mathrm{G}$ needle at 4 different sites. Medium (100 $\mu \mathrm{l})$ was also injected into animals of the sham group and the medium-treated group. At 4 weeks following transplantation, the rats were sacrificed for further studies.

Echocardiography. Cardiac function was measured 4 weeks following transplantation with an echocardiographic system equipped with a 12-MHz transducer (HP 5500; Hewlett-Packard, Palo Alto, CA, USA). LV end-diastolic diameter (LVEDD), LV end-systolic diameter, LV ejection fraction (LVEF) and fractional shortening (FS) were measured.

Histological analysis. To detect the cell survival rates following MSC implantation, prior to transplantation, transfected MSCs were also fluorescently labeled with DAPI (Beyotime Institute of Biotechnology, Haimen, China), according to the manufacturer's protocol. At 4 weeks following transplantation, the hearts ( $n=3$ each from the GFP-MSCs-treated group and ADM-MSCs-treated group) were dissected and embedded in optimum cutting temperature compound, snap-frozen in liquid nitrogen $\left(-196^{\circ} \mathrm{C}\right)$, and cut into $6-\mu \mathrm{m}$ sections. Transplanted cells that expressed green and blue fluorescence were observed by fluorescence microscopy.

To detect fibrosis in cardiac muscle, the hearts $(n=6$ from each group) were fixed in $10 \%$ formalin, cut transversely, embedded in paraffin, and stained with Masson's trichrome staining. The size of fibrotic areas was quantified as described (6). Transverse sections were randomly obtained from two levels, and 10 randomly selected fields per section were analyzed. After each field was scanned with a digital image analyzer, the area of the collagenous fraction was calculated as the sum of all areas containing connective tissue divided by the total area of the image.

Reverse transcription quantitative-polymerase chain reaction $(R T-q P C R)$. Total RNA was extracted from the cardiac muscle tissue using TRIzol reagent (Invitrogen; Thermo Fisher Scientific, Inc., Waltham, MA, USA), following the manufacturer's protocol. cDNA was generated using avian myeloblastosis virus reverse transcriptase (Promega Corporation, Madison, WI, USA) and oligo (dT) primers according to the manufacturer's instructions. Primer sequences and reaction conditions are listed in Table I. Real-time PCR was performed in an Applied Biosystems 7300 Fast Real-Time PCR System (Applied Biosystems; Thermo Fisher Scientific, Inc.) with SYBR Green PCR Master Mix (Bioneer Corporation, Daejeon, Korea). Primer sequences used and thermocycling conditions are presented in Table I. $\beta$-actin mRNA amplified from the same samples served as an internal control. The relative $\beta$-actin expression of each targeted gene was normalized by subtracting the corresponding threshold cycle $(\mathrm{Ct})$ values using the $2^{-\Delta \Delta \mathrm{Cq}}$ comparative method (14).

Western blotting. To identify the protein expression of ADM, matrix metalloproteinase (MMP)-2 and MMP-9, western blotting was performed. Cardiac tissues were lysed using radioimmunoprecipitation assay lysis buffer (Beyotime Institute of Biotechnology). A bicinchoninic acid protein assay kit (Beyotime Institute of Biotechnology) was used to determine protein concentrations. Protein extracts $(20 \mu \mathrm{g})$ were subjected to electrophoresis through an 8-15\% SDS-PAGE gel and were transferred to a polyvinylidene difluoride membrane. Following blocking with $5 \%$ skimmed milk at room temperature for $1 \mathrm{~h}$, the membrane was incubated with primary antibody at $37^{\circ} \mathrm{C}$ for 2 h: Anti-ADM (cat. no. BA0629; 1:200; Wuhan Boster Biological Technology, Ltd., Wuhan, China); anti-MMP-2 (cat. no. sc-13594; 1:200; Santa Cruz Biotechnology, Inc., Dallas, TX, USA); anti-MMP-9 (cat. no. BA2202; 1:200; Wuhan Boster Biological Technology, Ltd.); and anti- $\beta$-actin (cat. no. BM3873; 1:200; Wuhan Boster Biological Technology, Ltd.). The membrane was subsequently incubated at $37^{\circ} \mathrm{C}$ for an additional hour in an alkaline phosphatase-conjugated secondary antibody (cat. no. BM2007; 1:1,000; Wuhan Boster Biological Technology, Ltd.). Immunoreactive bands were visualized by enhanced chemiluminescence (Pierce; Thermo Fisher Scientific, Inc.) and exposure to X-ray film. The X-ray films were photographed using an Alpha Innotech gel analysis system (Bio-Rad Laboratories, Inc., Hercules, CA, USA). Relative protein expression was quantified using Quantity One software (version 4.62; Bio-Rad Laboratories, Inc.).

Statistical analysis. All values are expressed as mean values \pm standard error of the mean. Comparisons of parameters among groups were analyzed using the one-way analysis of variance followed by Tukey's post hoc test. $\mathrm{P}<0.05$ was considered to indicate a statistically significant difference. 


\section{Results}

Transduction of MSCs. To choose the optimal MOI, MSCs were transduced with the adenoviral vector at various MOIs. The results of observation by fluorescence microscopy indicated that most of the MSCs became round in shape and detached from the wall in 2 days at an MOI of 50, and that the percentage of GFP-positive cells was lower at an MOI of 400 . Therefore, adenoviral vectors were used to infect MSCs at an MOI of 200 in the subsequent experiments. As presented in Fig. 1A, most of these transfected cells were alive observed by light microscopy, and these cells presented green fluorescence observed by fluorescence microscopy. Furthermore, flow cytometric analysis also indicated that the transduction efficiency was $>90 \%$ (Fig. 1B). These results were confirmed by western blotting, which indicated that the expression of ADM protein was significantly higher in the ADM-MSCs than the GFP-MSCs (Fig. 1C and D).

Effect of cell transplantation on heart function. At 4 weeks following MSC transplantation, cardiac function of the living rats was measured by echocardiography. Results indicated that, compared with the sham group, the medium-treated group exhibited a significant decrease in LVEF and FS and an increase in LVEDD. These results demonstrated that the ISO injection could impair heart function in rats. When compared with the medium-treated group, cardiac function was significantly improved in both of the cell-treated groups. More importantly, LVEF and FS were noticeably higher in the ADM-MSCs-treated group than in the GFP-MSCs-treated group. Therefore, the authors can conclude that, compared with GFP-MSCs, ADM-MSC transplantation can further improve heart function (Fig. 2 and Table II).

Expression of ADM. At 4 weeks following MSC transplantation, the expression of ADM was examined by western blot analysis. Consistent with a previous study of the authors (7), the current study demonstrated that MSC transplantation could increase the expression of ADM in the myocardium. Furthermore, the present study also showed that ADM-MSCs transplantation could further increase the expression of ADM compared with GFP-MSCs transplantation (Fig. 3A and B).

Survival of transplanted cells. To observe the survival of MSCs in vivo, the nuclei of the transfected MSCs were also labeled with DAPI. As demonstrated in Fig. 4A and B, the survival of MSCs is higher in the ADM-MSCs-treated group than that in the GFP-MSCs-treated group.

Effect of cell transplantation on cardiac fibrosis. The extent of myocardial fibrosis was assessed by Masson's trichrome staining. Fig. 5A presents representative photographs of Masson's trichrome staining of heart sections 4 weeks following transplantation. Quantitative analysis of the fibrotic area was also performed. As identified in Fig. 5B, the fibrotic area was significantly greater in the medium-treated group than the GFP-MSCs-treated group and the ADM-MSCs-treated group. Furthermore, the fibrotic area of the ADM-MSCs-treated group was significantly smaller than that of the GFP-MSCs-treated group.
Table I. Primer sequences used.

\begin{tabular}{|c|c|c|c|}
\hline Primer & Direction & Sequence $\left(5^{\prime}-3^{\prime}\right)$ & $\mathrm{T}_{\mathrm{m}}\left({ }^{\circ} \mathrm{C}\right)$ \\
\hline MMP-2 & $\begin{array}{l}\text { Forward } \\
\text { Reverse }\end{array}$ & $\begin{array}{l}\text { aaggatcggtttatttggcg } \\
\text { cattccetgcgaagaacaca }\end{array}$ & 55 \\
\hline MMP-9 & $\begin{array}{l}\text { Forward } \\
\text { Reverse }\end{array}$ & $\begin{array}{l}\text { aagttctcgaatcacggaggaa } \\
\text { cattttcgcccagagaaga }\end{array}$ & 56 \\
\hline HGF & $\begin{array}{l}\text { Forward } \\
\text { Reverse }\end{array}$ & $\begin{array}{l}\text { ttgtgagggagattatggtggc } \\
\text { caggacgatttgggatggcatag }\end{array}$ & 57 \\
\hline TGF- $\beta 1$ & $\begin{array}{l}\text { Forward } \\
\text { Reverse }\end{array}$ & $\begin{array}{l}\text { caacaattcctggcgtta } \\
\text { aagccetgtattccgtctcc }\end{array}$ & 56 \\
\hline Collagen-1 & $\begin{array}{l}\text { Forward } \\
\text { Reverse }\end{array}$ & $\begin{array}{l}\text { cagattgggatggagggagttta } \\
\text { ctacagcacgcttgtggatggct }\end{array}$ & 56 \\
\hline Collagen-III & $\begin{array}{l}\text { Forward } \\
\text { Reverse }\end{array}$ & $\begin{array}{l}\text { atagctgaactgaaagccaccat } \\
\text { cctgaactcaagagcggaata }\end{array}$ & 55 \\
\hline$\beta$-actin & $\begin{array}{l}\text { Forward } \\
\text { Reverse }\end{array}$ & $\begin{array}{l}\text { cccatctatgagggttacgc } \\
\text { tttaatgtcacgcacgatttc }\end{array}$ & 56 \\
\hline
\end{tabular}

Thermocycling parameters: pre-denaturation $5 \mathrm{~min}$ at $95^{\circ} \mathrm{C}, 40$ cycles of $10 \mathrm{sec}$ at $95^{\circ} \mathrm{C}, 30 \mathrm{sec}$ at $55-57^{\circ} \mathrm{C}$ and melting at $65-90^{\circ} \mathrm{C}, 1^{\circ} \mathrm{C}$ for $1 \mathrm{sec}$. MMP, matrix metalloproteinase; HGF, hepatocyte growth factor; TGF- $\beta 1$, transforming growth factor- $\beta$.

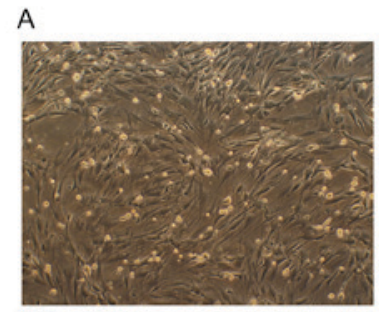

B

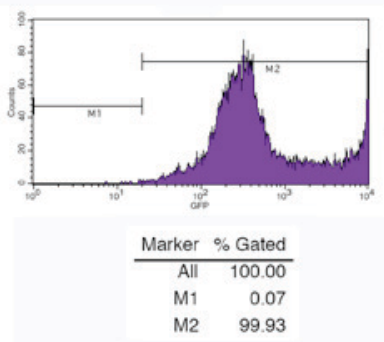

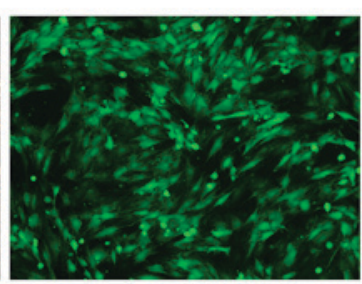

C

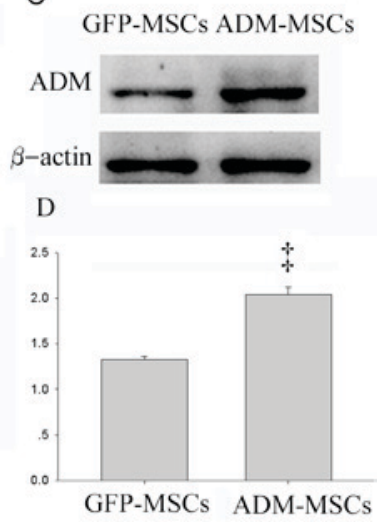

Figure 1. Transfection of MSCs. (A) Transfected MSCs observed by light and fluorescent microscopy (multiplicity of infection, 1:200; magnification, x40). (B) Transduction efficiency determined by fluorescence analysis. (C) Western blot analysis of ADM protein in ADM-MSCs and GFP-MSCs (D) Quantitative analysis of western blot results. ${ }^{\mathrm{P}}<0.01$ vs. the GFP-MSCs. Data are presented as the mean \pm standard error of the mean. MSCs, mesenchymal stem cells; ADM, adrenomedullin; GRP, green fluorescent protein.

Next, the expression of collagen I and III were investigated by RT-qPCR. Similar to the authors' previous study (6), the present study indicated that, compared with the sham group, the expressions of collagen I and III were significantly higher 
Table II. Echocardiographic assessment of cardiac function.

\begin{tabular}{lccccc}
\hline Group & $\mathrm{n}$ & LVDd $(\mathrm{mm})$ & LVDs $(\mathrm{mm})$ & EF $(\%)$ & FS $(\%)$ \\
\hline Sham & 9 & $6.39 \pm 0.44$ & $3.16 \pm 0.22$ & $86.503 \pm 1.54$ & $50.66 \pm 1.87$ \\
Medium-treated & 8 & $7.25 \pm 1.69$ & $5.35 \pm 1.57^{\mathrm{a}}$ & $56.67 \pm 6.86^{\mathrm{b}}$ & $26.27 \pm 4.01^{\mathrm{b}}$ \\
GFP-MSCs-treated & 9 & $6.671 \pm 0.70$ & $4.24 \pm 0.92^{\mathrm{c}}$ & $69.235 \pm 7.30^{\mathrm{b}, \mathrm{c}}$ & $34.59 \pm 5.13^{\mathrm{b}, \mathrm{c}}$ \\
ADM-MSCs-treated & 9 & $6.696 \pm 0.81$ & $3.95 \pm 0.55^{\mathrm{c}}$ & $79.403 \pm 1.70^{\mathrm{b}-\mathrm{d}}$ & $43.48 \pm 2.15^{\mathrm{b}-\mathrm{d}}$ \\
\hline
\end{tabular}

${ }^{\mathrm{a}} \mathrm{P}<0.01$ vs. sham group; ${ }^{\mathrm{P}}<0.05$ vs. sham group; ${ }^{\mathrm{c}} \mathrm{P}<0.05$ vs. medium-treated group; ${ }^{\mathrm{d}} \mathrm{P}<0.05$ vs. GFP-MSCs-treated group. Comparisons of parameters among groups were analyzed using analysis of variance. GFP, green fluorescence protein; MSCs, mesenchymal stem cells; ADM, adrenomedullin; EF, ejection fraction; FS, fractional shortening; LVDd, left ventricular diastolic diameter; LVDs, left ventricular systolic diameter.

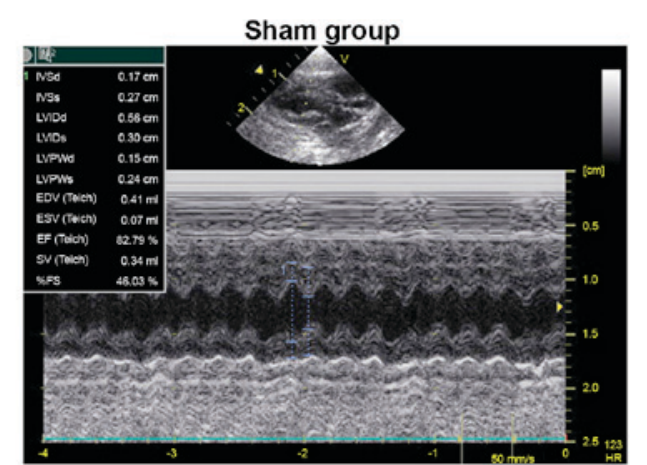

GFP-MSCs-treated group

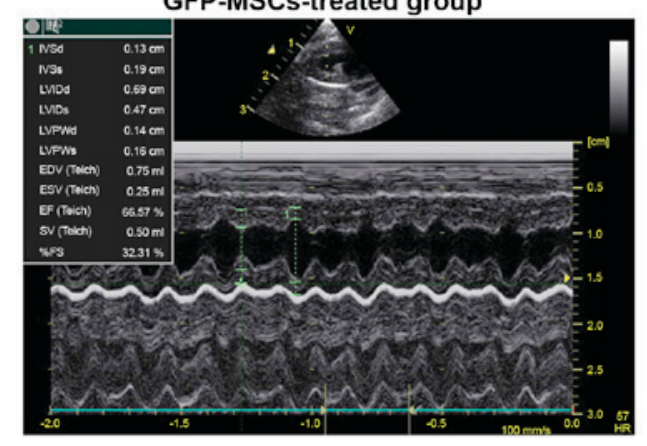

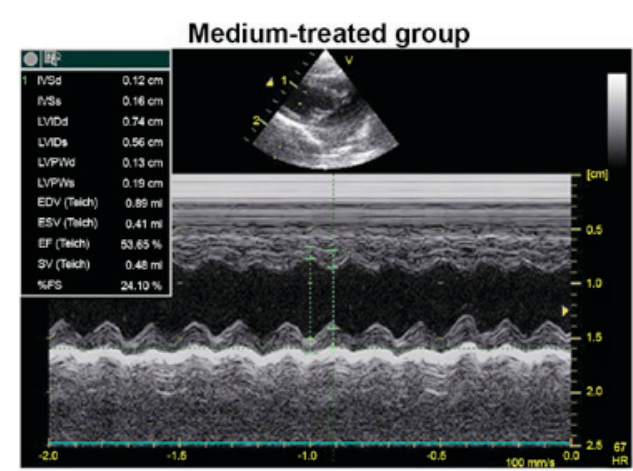

ADM-MSCs-treated group

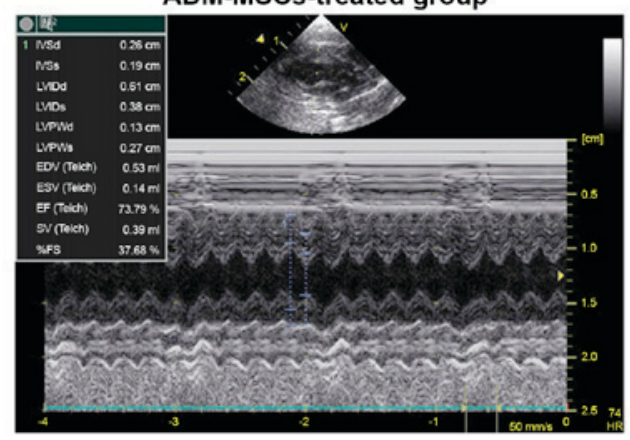

Figure 2. Representative echocardiography images for all four experimental groups. GFP, green fluorescent protein; ADM, adrenomedullin; MSCs, mesenchymal stem cells.

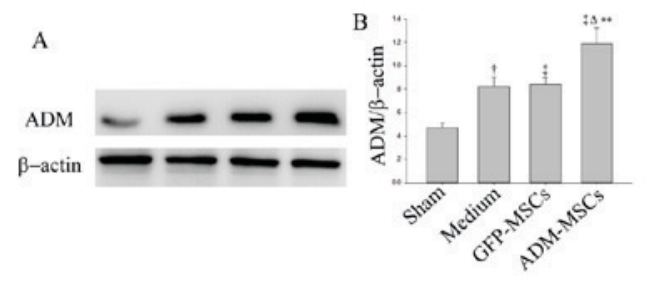

Figure 3. Expression of ADM. (A) The expression of AMD was measured by western blotting. (B) Densitometric analysis was used to calculate the relative ratio of $A D M / \beta$-actin. Data are presented as the mean \pm standard error of the mean. ${ }^{* *} \mathrm{P}<0.01$ vs. the GFP-MSCs-treated group, ${ }^{\wedge} \mathrm{P}<0.05$ vs. the Medium-treated group, ${ }^{\circ} \mathrm{P}<0.05$ vs. the Sham group, ${ }^{\circ} \mathrm{P}<0.01$ vs. the Sham group. MSCs, mesenchymal stem cells; ADM, adrenomedullin; GRP, green fluorescent protein

in the medium-treated group. In addition, cell transplantation significantly decreased the expression of collagen I and III. Importantly, the expressions of collagen I and III were further decreased in the ADM-MSCs-treated group compared with the GFP-MSCs-treated group (Fig. 6).

Effect of cell transplantation on expression of MMPs. The authors investigated the expression of MMP-2 and MMP-9 by RT-qPCR and western blotting. The results demonstrated that, compared with the medium-treated group, the gene expression of MMP-2 and MMP-9 was significantly lower in both of the cell treated groups. Importantly, compared with the GFP-MSCs-treated group, the ADM-MSCs-treated group exhibited even lower expression of MMP-2 (Fig. 7A). In addition, the groups treated with both cell types had significantly lower MMP-2 and MMP-9 protein levels compared with the medium-treated group. The protein level of MMP-2 was also lower in the ADM-MSCs-treated group than the GFP-MSCs-treated group (Fig. 7B and C). Although the gene and protein expression of MMP-9 were lower in the ADM-MSCs-treated group than that in the GFP-MSCs-treated 

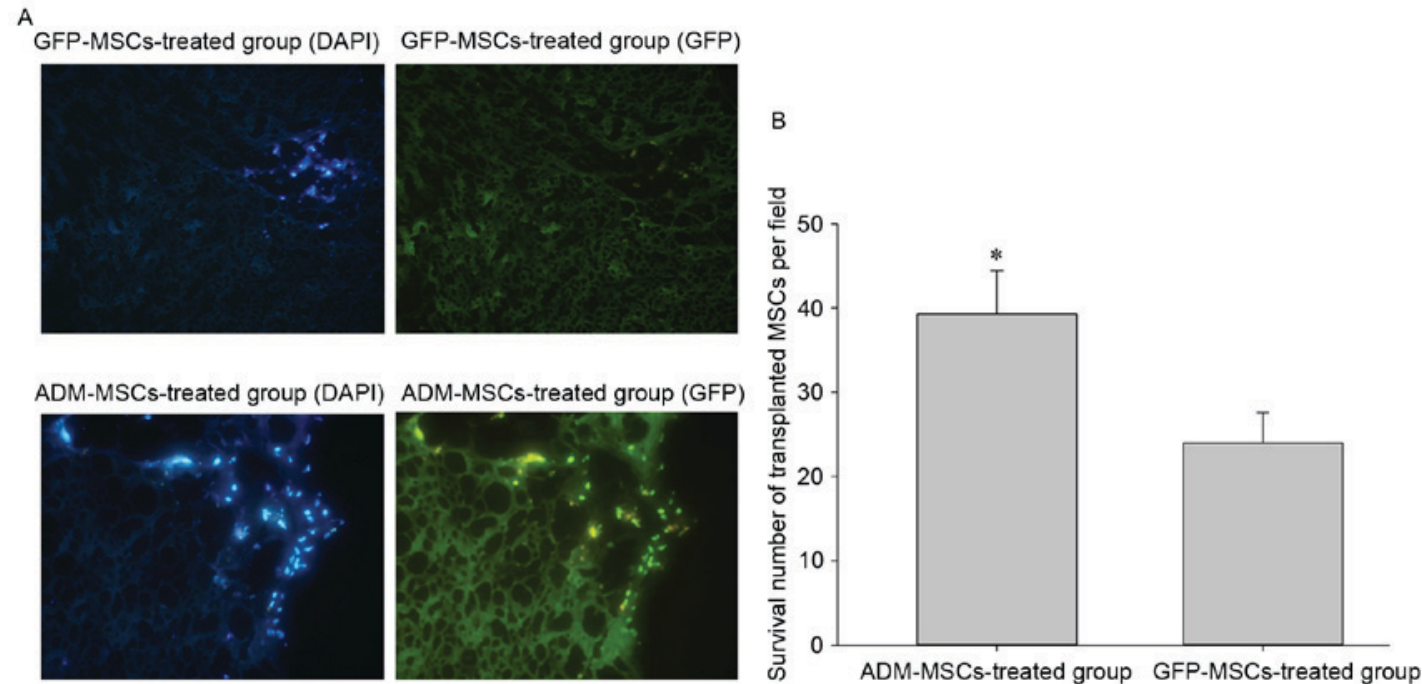

Figure 4. Survival of transplanted MSCs. (A) Representative frozen myocardial sections. (B) Data are presented as the mean \pm standard error of the mean. ${ }^{*} \mathrm{P}<0.05$ vs. the GFP-MSCs-treated group. MSCs, mesenchymal stem cells; ADM, adrenomedullin; GRP, green fluorescent protein.
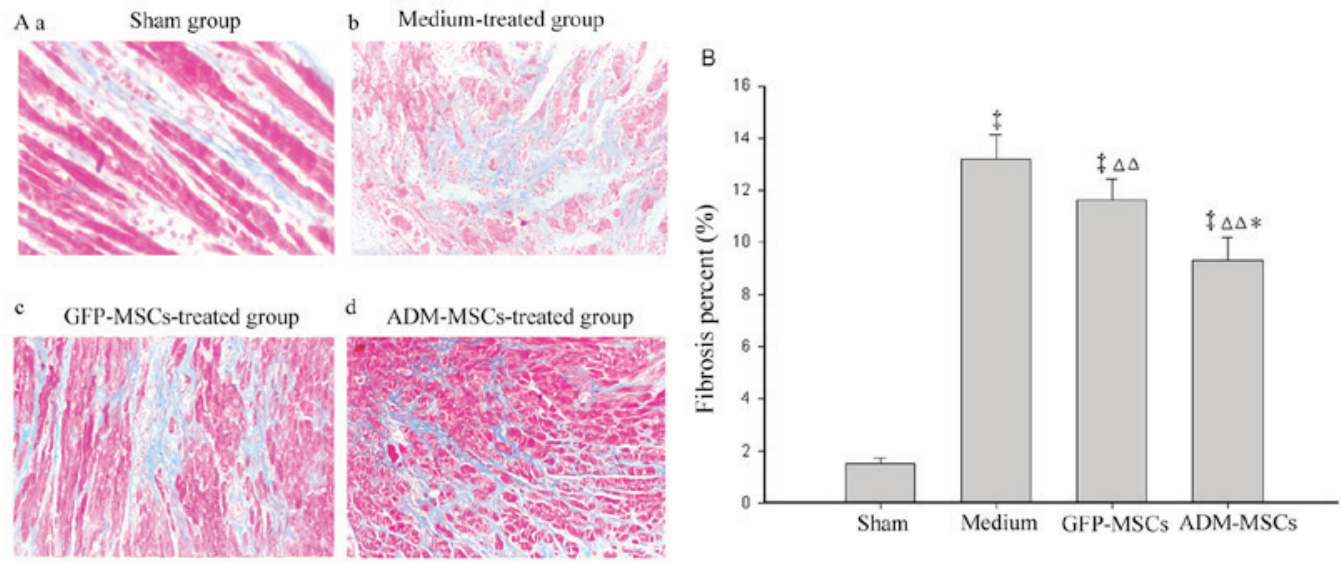

Figure 5. Effect of cell transplantation on cardiac fibrosis. (A) Representative myocardial sections stained with Masson's trichrome staining of four groups (magnification, x200): (a) Sham group, (b) medium-treated group, (c) GRP-MSCs-treated group and the (d) ADM-MSCs-treated group. (B) Quantitative analysis of collagen fraction. ${ }^{*} \mathrm{P}<0.05$ vs. the GFP-MSCs-treated group, ${ }^{\Delta \Delta} \mathrm{P}<0.01$ vs. the Medium-treated group, ${ }^{+} \mathrm{P}<0.01$ vs. the Sham group. MSCs, mesenchymal stem cells; ADM, adrenomedullin; GRP, green fluorescent protein.
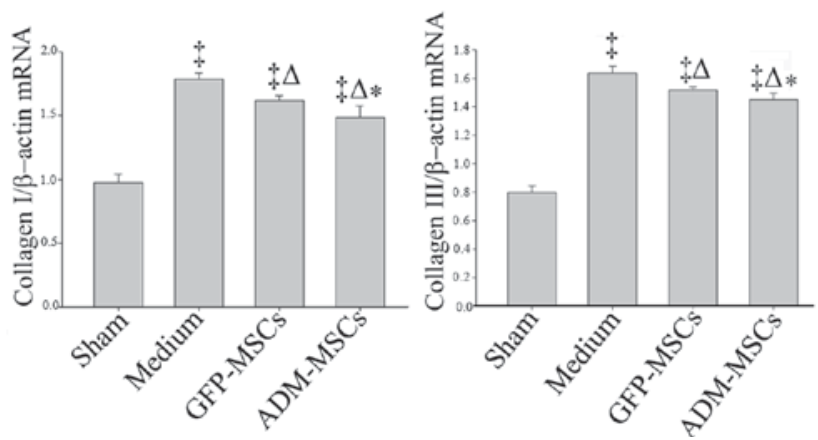

Figure 6. Reverse transcription-quantitative polymerase chain reaction analysis of collagen I and collagen III mRNA levels. ${ }^{*} \mathrm{P}<0.05$ vs. the GFP-MSCs-treated group, ${ }^{\Delta} \mathrm{P}<0.05$ vs. the Medium-treated group, ${ }^{\stackrel{ }{ }} \mathrm{P}<0.01$ vs. the Sham group. MSCs, mesenchymal stem cells; ADM, adrenomedullin; GRP, green fluorescent protein.

group, the difference between these two groups was not statistically significant.
Paracrine signaling by cell transplantation. The expression of the hepatocyte growth factor (HGF) and transforming growth factor (TGF)- $\beta$ genes, which mediate cardiac remodeling, were then investigated. As presented in Fig. 7A, compared with the expression in the medium-treated group, the gene expression of HGF was significantly lower in both the GFP-MSCs-treated group and the ADM-MSCs-treated group. Importantly, compared with the GFP-MSCs-treated group, the ADM-MSCs-treated group exhibited lower levels of HGF gene expression. In addition, it was also indicated that, compared with the GFP-MSCs-treated group, the ADM-MSCs-treated group had not lower levels of TGF- $\beta$ gene expression (Fig. 7A).

\section{Discussion}

In the present article, the authors demonstrated that, compared to the GFP-MSCs transplantation, ADM-MSC transplantation greatly improved cardiac function and decreased the cardiac fibrosis associated with ISO-induced heart failure. In addition, 

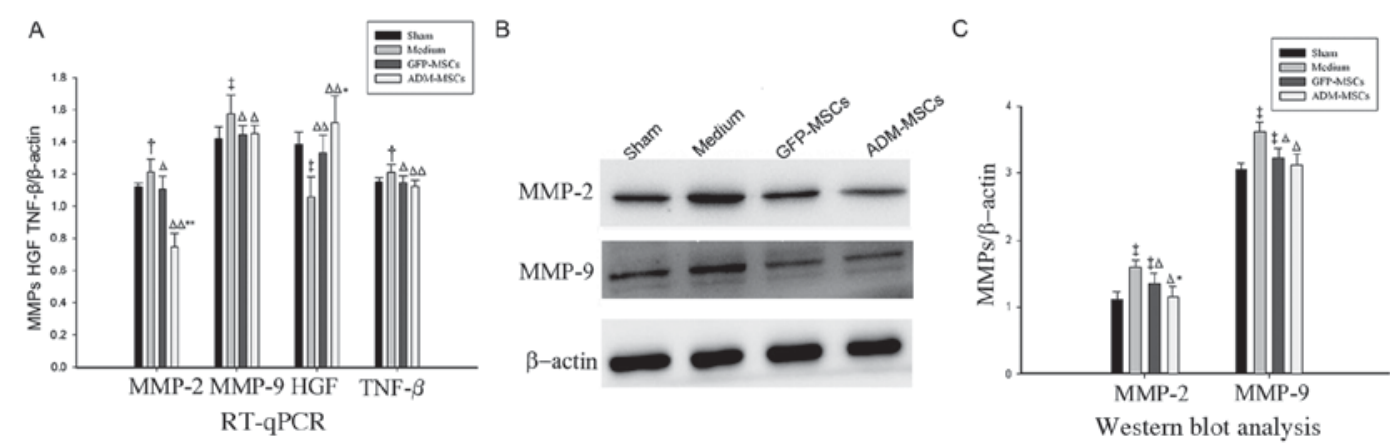

Figure 7. (A) RT-qPCR analysis of mRNA levels. (B and C) Western blot analysis of protein levels. ${ }^{*} \mathrm{P}<0.05,{ }^{* *} \mathrm{P}<0.01$ vs. the GFP-MSCs-treated group; ${ }^{\Delta} \mathrm{P}<0.05,{ }^{\Delta} \mathrm{P}<0.01$ vs. the Medium-treated group; ${ }^{\mathrm{P}} \mathrm{P}<0.05,{ }^{\dagger} \mathrm{P}<0.01$ vs. the Sham group. MMP, matrix metalloproteinase; HGF, hepatocyte growth factor; TNF- $\beta$, tumor necrosis factor- $\beta$; RT-qPCR, reverse transcription-quantitative polymerase chain reaction.

the beneficial effects of ADM-MSC transplantation may be partially mediated by significantly influencing the expression of fibrosis-related cytokines, such as ADM and HGF.

Studies have reported that subcutaneous administration of ISO in rats could produce myocardial cell death and cardiac fibrosis and lead to heart failure, while maintaining an intact coronary vasculature $(15,16)$. The effectiveness of reperfusion therapy and the potential confounding effects of an abnormal blood supply underscore the importance of studying cardiac fibrosis arising in the presence of an intact vascular system (16). For these reasons, the ISO-treated rat heart is an excellent model system for investigations into cardiac fibrosis and heart failure. Previous studies, including the authors' published study, have proven that ISO may induce heart failure and cardiac fibrosis 4 weeks subsequent to injection $(6,16,17)$. Additionally, it is well-known that cardiac fibrosis may have already formed in patients with previous myocardial infarction and dilated cardiomyopathy (18). Therefore, in order to investigate whether cell transplantation can inhibit the progress of cardiac fibrosis of these patients, the authors chose the interval of 4 weeks following ISO injection to initiate MSC transplantation.

Paracrine mechanisms have been proven to serve an important role in the transplantation of MSCs. The authors' previous study had also indicated that MSCs could secrete ADM and that the level of ADM in the myocardium significantly increased after the transplantation of MSCs, coinciding with the decrease in cardiac fibrosis (6). Therefore, it was concluded that ADM secreted by the MSCs may play an important role in MSC transplantation. ADM has been reported to inhibit myocardial remodeling by attenuating cardiomyocyte hypertrophy, the proliferation of myocardial fibroblasts and the production of extracellular matrix (10-13). Thus, in the current study, the authors sought to investigate whether, compared with MSCs alone, ADM gene-modified MSCs can further decrease cardiac fibrosis and increase heart function.

In the present study, GFP-MSCs, ADM-MSCs or medium were grafted into the myocardium in a rat model of ISO-induced heart failure. At 4 weeks following transplantation, heart function was examined, and the results showed that, compared with GFP-MSCs and medium, ADM-MSCs could significantly improve heart function, as indicated by an increase in LVEF. Therefore, it may be concluded that modifying MSCs with ADM can increase the beneficial effects of MSC transplantation.
Cardiac fibrosis serves an important role in the pathogenesis of heart failure. Many previous studies have shown that inhibiting the development of cardiac fibrosis could lead to the improvement of heart function $(19,20)$. A previous study reported that MSCs transplantation could improve heart function partly by inhibiting myocardial fibrosis (6). Furthermore, ADM have been proven to play a role as an autocrine/paracrine modulator in the process of cardiac remodeling by suppressing mitogenesis and collagen synthesis in fibroblasts (11). Therefore, the effect of ADM modified MSCs on cardiac fibrosis were investigated in the study. Histological and molecular analysis demonstrated that transplantation of ADM-MSCs could result in the greatest decrease in cardiac fibrosis, compared with medium transplantation and GFP-MSC transplantation.

The MMPs are a family of $>25$ species of zinc-dependent proteases, which have been shown to be important in ventricular remodeling by degrading the extracellular matrix. In the failing heart, the normal collagens are degraded by increased levels of MMPs, and fibrous interstitial deposits of poorly cross-linked collagens are synthesized. This, in turn, may lead to myocardial fibrosis, dilation of the ventricles and cardiac dysfunction $(21,22)$. Inhibition of the activities of the MMPs prevents progressive left ventricular remodeling and improves heart function in an animal model of heart failure $(23,24)$. Regardless of the inciting cause, there appears to be increased expression of MMPs in the initial phase and the final phase of heart failure, leading to marked ventricular dilation and fibrosis $(25,26)$. The authors' previous results have indicated that the expression of MMP-2 and MMP-9 was significantly increased in ISO-induced heart failure (6). In addition, ADM has been proven to have an ability to inhibit the expression of MMP-2 and MMP-1 in hepatic stellate cells and rheumatoid synovial fibroblasts $(27,28)$. Therefore, the effect of ADM-MSC transplantation on the expression of MMP-2 and MMP-9 was investigated in the current study. Importantly, the results also showed that, compared with the GFP-MSCs-treated group, the ADM-MSCs-treated groups exhibited a significant decrease in MMP-2 levels. Therefore, the authors propose that compared with MSCs, ADM gene-modified MSCs can decrease the expression of MMP-2 significantly, resulting in a greater decrease in the degradation of normal collagen and greater improvement in heart function. 
Next, the effect of ADM on the paracrine ability of MSCs was explored. The expression of ADM in the myocardium was investigated. Results demonstrated that the level of ADM was significantly increased in the ADM-MSCs-treated group than that of the GFP-MSCs-treated group, which is coinciding with the decrease of myocardial fibrosis. Therefore, it may be concluded that ADM-MSCs can inhibit myocardial fibrosis by secreting ADM. Furthermore, HGF (29) and TGF- $\beta$ (30), which is related to myocardial fibrosis, were investigated. The authors' previous study had indicated that the transplantation of MSCs increased the expression of HGF in the myocardium (6). In the present study, results demonstrated that the ADM-MSCs-treated group exhibited a significant increase in HGF, when compared with the GFP-MSCs-treated and medium-treated groups. The expression of TGF- $\beta$ was also observed. However, these results presented no significant difference between the ADM-MSCs-treated group and the GFP-MSCs-treated group. These observations suggested that ADM-MSCs may decrease myocardial fibrosis not only by secreting ADM, but by changing the expression of fibrosis-related cytokines, such as HGF. Further studies will be required to detect the mechanism by which the ADM-MSCs influence the expression of these fibrosis-related cytokines. In addition, the current study demonstrated that ADM modification could increase the survival of transplanted cells.

In conclusion, the transplantation of ADM-MSCs can greatly improve cardiac function by attenuating myocardial fibrosis. The beneficial effect of ADM-MSC transplantation may be partially mediated through changes in the expression of fibrosis-related genes, such as ADM and HGF.

\section{Acknowledgements}

The authors would like to thank Dr Wei Liu for her expert assistance with cell culture and western blot analysis. Dr Wei Liu is a member of the Key Laboratory of Myocardial Ischemia Mechanism and Treatment, Harbin Medical University (Harbin, China). The present study was supported by grants from the Natural Science Foundation of Heilongjiang Province (grant no. QC2013C108).

\section{References}

1. Sun R, Li X, Liu M, Zeng Y, Chen S and Zhang P: Advances in stem cell therapy for cardiovascular disease (Review). Int J Mol Med 38: 23-29, 2016.

2. Zhang C, Nong Y, Tong S, Yao Q, Wen L, Zhang Z, Wei L, Cheng J, Feng Y and Song Z: Triptolide improves early survival of mesenchymal stem cells transplantation into rat myocardium. Cardiology 128: 73-85, 2014.

3. Choi SH, Jung SY, Kwon SM and Baek SH: Perspectives on stem cell therapy for cardiac regeneration: Advances and challenges. Circ J 76: 1307-1312, 2012.

4. Chin SP, Poey AC, Wong CY, Chang SK, Tan CS, Ng MT, Chew KH, Lam KH and Cheong SK: Intramyocardial and intracoronary autologous bone marrow-derived mesenchymal stromal cell treatment in chronic severe dilated cardiomyopathy. Cytotherapy 13: 814-821, 2011.

5. Suresh SC, Selvaraju V, Thirunavukkarasu M, Goldman JW, Husain A, Alexander Palesty J, Sanchez JA, McFadden DW and Maulik N: Thioredoxin-1 (Trx1) engineered mesenchymal stem cell therapy increased pro-angiogenic factors, reduced fibrosis and improved heart function in the infracted rat myocardium. Int J Cardiol 201: 517-528, 2015.
6. Li L, Zhang Y, Li Y, Yu B, Xu Y,Zhao S and Guan Z: Mesenchymal stem cell transplantation attenuates cardiac fibrosis associated with isoproterenol-induced global heart failure. Transpl Int 21: 1181-1189, 2008 .

7. Li L, Zhang S, Zhang Y, Yu B, Xu Y and Guan Z: Paracrine action mediate the antifibrotic effect of transplanted mesenchymal stem cells in a rat model of global heart failure. Mol Biol Rep 36: 725-731, 2009.

8. Xu B, Luo Y, Liu Y, Li BY and Wang Y: Platelet-derived growth factor-BB enhances MSC-mediated cardioprotection via suppression of miR-320 expression. Am J Physiol Heart Circ Physiol 308: H980-H989, 2015.

9. Wen Z, Huang W, Feng Y, Cai W, Wang Y, Wang X, Liang J, Wani M, Chen J, Zhu P, et al: MicroRNA-377 regulates mesenchymal stem cell-induced angiogenesis in ischemic hearts by targeting VEGF. PLoS One 9: e104666, 2014.

10. Tsuruda T, Kato J, Kitamura K, Kawamoto M, Kuwasako K, Imamura T, Koiwaya Y, Tsuji T, Kangawa K and Eto T: An autocrine or a paracrine role of adrenomedullin in modulating cardiac fibroblast growth. Cardiovasc Res 43: 958-967, 1999.

11. Horio T, Nishikimi T, Yoshihara F, Matsuo H, Takishita S and Kangawa K: Effects of adrenomedullin on cultured rat cardiac myocytes and fibroblasts. Eur J Pharmacol 382: 1-9, 1999.

12. Nishikimi T, Yoshihara F, Mori Y, Kangawa K and Matsuoka H: Cardioprotective effect of adrenomedullin in heart failure. Hypertens Res 26 (Suppl): S121-S127, 2003.

13. Okumura H, Nagaya $\mathrm{N}$ and Kangawa K: Adrenomedullin infusion during ischemia/reperfusion attenuates left ventricular remodeling and myocardial fibrosis in rats. Hypertens Res 26 (Suppl): S99-S104, 2003.

14. Livak KJ and Schmittgen TD: Analysis of relative gene expression data using real-time quantitative PCR and the 2(-Delta Delta C(T)) method. Methods 25: 402-408, 2001.

15. Okuda N, Hayashi T, Mori T, Inamoto S, Okabe M, Mieno S, Horimoto $\mathrm{H}$ and Kitaura Y: Nifedipine enhances the cardioprotective effect of an angiotensin-II receptor blocker in an experimental animal model of heart failure. Hypertens Res 28: 431-438, 2005.

16. Teerlink JR, Pfeffer JM and Pfeffer MA: Progressive ventricular remodeling in response to diffuse isoproterenol-induced myocardial necrosis in rats. Circ Res 75: 105-113, 1994.

17. Brouri F, Hanoun N, Mediani O, Saurini F, Hamon M, Vanhoutte PM and Lechat P: Blockade of beta1- and desensitization of beta 2-adrenoceptors reduce isoprenaline-induced cardiac fibrosis. Eur J Pharmacol 485: 227-234, 2004.

18. Maisch B: Ventricular remodeling. Cardiology 87 (Suppl 1): S2-S10, 1996.

19. Eckhouse SR and Spinale FG: Changes in the myocardial interstitium and contribution to the progression of heart failure. Heart Fail Clin 8: 7-20, 2012.

20. Deschamps AM and Spinale FG: Matrix modulation and heart failure: New concepts question old beliefs. Curr Opin Cardiol 20: 211-216, 2005.

21. Spinale FG: Myocardial matrix remodeling and the matrix metalloproteinases: Influence on cardiac form and function. Physiol Rev 87: 1285-1342, 2007.

22. Vanhoutte D, Schellings M, Pinto Y and Heymans S: Relevance of matrix metalloproteinases and their inhibitors after myocardial infarction: A temporal and spatial window. Cardiovasc Res 69: 604-613, 2006

23. Song YH, Cai H, Gu N, Qian CF, Cao SP and Zhao ZM: Icariin attenuates cardiac remodelling through down-regulating myocardial apoptosis and matrix metalloproteinase activity in rats with congestive heart failure. J Pharm Pharmacol 63: 541-549, 2001.

24. Creemers EE, Cleutjens JP, Smits JF and Daemen MJ: Matrix metalloproteinase inhibition after myocardial infarction: A new approach to prevent heart failure? Circ Res 89: 201-210, 2001.

25. Li YY, McTiernan CF and Feldman AM: Interplay of matrix metalloproteinases, tissue inhibitors of metalloproteinases and their regulators in cardiac matrix remodeling. Cardiovasc Res 46: 214-224, 2000.

26. Boixel C, Fontaine V, Rücker-Martin C, Milliez P, Louedec L, Michel JB, Jacob MP and Hatem SN: Fibrosis of the left atria during progression of heart failure is associated with increased matrix metalloproteinases in the rat. J Am Coll Cardiol 42: 336-344, 2003.

27. Wang Y, Zhang JS, Qian J, Huang GC and Chen Q: Adrenomedullin regulates expressions of transforming growth factor-beta1 and beta1-induced matrix metalloproteinase-2 in hepatic stellate cells. Int J Exp Pathol 87: 177-184, 2006. 
28. Lee EG, Lee S, Chae HJ, Park SJ, Lee YC and Yoo WH: Adrenomedullin inhibits IL-1 $\beta$-induced rheumatoid synovial fibroblast proliferation and MMPs, COX-2 and PGE2 production. Inflammation 34: 335-343, 2011.

29. Li Y, Takemura G, Kosai K, Yuge K, Nagano S, Esaki M, Goto K, Takahashi T, Hayakawa K, Koda M, et al: Postinfarction treatment with an adenoviral vector expressing hepatocyte growth factor relieves chronic left ventricular remodeling and dysfunction in mice. Circulation 107: 2499-2506, 2003.
30. Edgley AJ, Krum H and Kelly DJ: Targeting fibrosis for the treatment of heart failure: A role for transforming growth factor- $\beta$. Cardiovasc Ther 30: e30-e40, 2012.

cc) (7) (8) This work is licensed under a Creative Commons Attribution 4.0 International (CC BY-NC 4.0) License 